\title{
Functional Imaging of Human Epidermal Growth Factor Receptor 2-Positive Metastatic Breast Cancer Using ${ }^{64} \mathrm{Cu}$-DOTA-Trastuzumab PET
}

Joanne E. Mortimer ${ }^{1}$, James R. Bading ${ }^{2}$, David M. Colcher ${ }^{2}$, Peter S. Conti ${ }^{3}$, Paul H. Frankel ${ }^{4}$, Mary I. Carroll ${ }^{1}$, Shan Tong ${ }^{2}$, Erasmus Poku ${ }^{2}$, Joshua K. Miles², John E. Shively ${ }^{5}$, and Andrew A. Raubitschek ${ }^{2}$

${ }^{I}$ Department of Medical Oncology and Experimental Therapeutics, City of Hope, Duarte, California; ${ }^{2}$ Department of Cancer Immunotherapeutics and Tumor Immunology, Beckman Research Institute of the City of Hope, Duarte, California; ${ }^{3}$ Molecular Imaging Center, Department of Radiology, University of Southern California, Los Angeles, California; ${ }^{4}$ Department of Information Sciences, City of Hope, Duarte, California; and ${ }^{5}$ Department of Immunology, Beckman Research Institute of the City of Hope, Duarte, California

Women with human epidermal growth factor receptor 2 (HER2)positive breast cancer are candidates for treatment with the antiHER2 antibody trastuzumab. Assessment of HER2 status in recurrent disease is usually made by core needle biopsy of a single lesion, which may not represent the larger tumor mass or other sites of disease. Our long-range goal is to develop PET of radiolabeled trastuzumab for systemically assessing tumor HER2 expression and identifying appropriate use of anti-HER2 therapies. The purpose of this study was to evaluate PET/CT of ${ }^{64} \mathrm{Cu}$-DOTA-trastuzumab for detecting and measuring tumor uptake of trastuzumab in patients with HER2-positive metastatic breast cancer. Methods: Eight women with biopsy-confirmed HER2-positive metastatic breast cancer and no anti-HER2 therapy for 4 mo or longer underwent complete staging, including ${ }^{18} \mathrm{~F}-\mathrm{FDG}$ PET/CT. For 6 of the 8 patients, ${ }^{64} \mathrm{Cu}$-DOTAtrastuzumab injection (364-512 MBq, $5 \mathrm{mg}$ of trastuzumab) was preceded by trastuzumab infusion $(45 \mathrm{mg})$. PET/CT (PET scan duration $1 \mathrm{~h}$ ) was performed 21-25 (day 1) and 47-49 (day 2) $\mathrm{h}$ after ${ }^{64} \mathrm{Cu}$-DOTA-trastuzumab injection. Scan fields of view were chosen on the basis of ${ }^{18} \mathrm{~F}-\mathrm{FDG} \mathrm{PET} / \mathrm{CT}$. Tumor detection sensitivity and uptake analyses were limited to lesions identifiable on CT; lesions visualized relative to adjacent tissue on PET were considered PETpositive. Radiolabel uptake in prominent lesions was measured as maximum single-voxel standardized uptake value $\left(S_{U} V_{\text {max }}\right)$. Results: Liver uptake of ${ }^{64} \mathrm{Cu}$ was reduced approximately $75 \%$ with the $45-\mathrm{mg}$ trastuzumab predose, without significant effect on tumor uptake. The study included 89 CT-positive lesions. Detection sensitivity was $77 \%$, $89 \%$, and $93 \%$ for day 1 , day 2 , and ${ }^{18} \mathrm{~F}-\mathrm{FDG}$, respectively. On average, tumor uptake was similar for ${ }^{64} \mathrm{Cu}$-DOTA-trastuzumab and ${ }^{18} \mathrm{~F}$-FDG (SUV $_{\text {max }}$ and range, 8.1 and 3.0-22.5 for day 1 [ $n=48$ ]; 8.9 and 0.928.9 for day 2 [ $n=38$ ]; 9.7 and 3.3-25.4 for ${ }^{18}$ F-FDG $\left.[n=56]\right)$, but same-lesion SUV $_{\max }$ was not correlated between the 2 radiotracers. No toxicities were observed, and estimated radiation dose from ${ }^{64} \mathrm{Cu}$-DOTA-trastuzumab was similar to ${ }^{18} \mathrm{~F}-\mathrm{FDG}$. Conclusion: ${ }^{64} \mathrm{Cu}$-DOTA-trastuzumab visualizes HER2-positive metastatic breast cancer with high sensitivity and is effective in surveying disseminated disease. A 45-mg trastuzumab predose provides a ${ }^{64} \mathrm{Cu}$-DOTA-trastuzumab biodistribution favorable for tumor imaging. ${ }^{64} \mathrm{Cu}$-DOTA-trastuzumab PET/CT warrants further evaluation

Received Mar. 4, 2013; revision accepted Jul. 9, 2013.

For correspondence or reprints contact: Joanne E. Mortimer, Department of Medical Oncology and Experimental Therapeutics, City of Hope National Medical Center, 1500 East Duarte Rd., Duarte, CA 91010.

E-mail: jmortimer@coh.org

Published online Dec. 12, 2013.

COPYRIGHT (c) 2014 by the Society of Nuclear Medicine and Molecular Imaging, Inc. for assessing tumor HER2 expression and individualizing treatments that include trastuzumab.

Key Words: ${ }^{64} \mathrm{Cu}$-labeled trastuzumab; HER2; breast cancer

J Nucl Med 2014; 55:23-29

DOI: 10.2967/jnumed.113.122630

$\mathbf{O}$ verexpression of human epidermal growth factor receptor 2 (HER2) is identified in $20 \%$ of breast cancers (1). Women with HER2-positive breast cancer are candidates for treatment with the humanized anti-HER2 antibody trastuzumab. When combined with chemotherapy, trastuzumab increases overall survival for all stages of HER2-positive breast cancer.

Trastuzumab is used in newly diagnosed HER2-positive breast cancer as adjuvant or neoadjuvant therapy and as treatment for metastatic disease at presentation or relapse if more than 6 mo since adjuvant trastuzumab. Response rates are at best about $25 \%$ and $50 \%$, respectively, for first-line trastuzumab and trastuzumab plus chemotherapy (2). Furthermore, in the adjuvant setting, patients classified as HER2-negative sometimes benefit (3). There is a clear need to better identify those patients who may benefit from these costly and potentially toxic therapies.

The accurate and comprehensive assessment of tumor HER2 status is critical in determining treatment. However, the pathologic assessment of HER2 status suffers from interlaboratory discordance and lack of a clear definition of positivity (4). Furthermore, confirmation of recurrent disease is usually made by core needle biopsy of an accessible lesion and may not represent the larger tumor mass or other sites of disease. Differences in HER2 expression between primary and metastatic tumors have been observed in as many as $20 \%$ of patients, especially when metastasis occurs after adjuvant or neoadjuvant therapy (5-7).

We hypothesized that assessment of tumor HER2 status can be improved by supplementing pathologic evaluation with functional imaging of HER2. Radiolabeled trastuzumab has been used to image patients with HER2-positive breast cancer, initially with ${ }^{111} \mathrm{In}$ and single-photon imaging $(8,9)$ and more recently with ${ }^{89} \mathrm{Zr}$ and PET (10). Although labeling with the positron-emitting isotope ${ }^{124} \mathrm{I}$ is also a possibility, radiometals are preferred given the known cellular internalization of trastuzumab and subsequent rapid efflux of radiolabel from cells when trastuzumab is labeled with 
isotopes of iodine (11). Tumor visualization has been variable, perhaps because the women were on active trastuzumab treatment, which may have inhibited radiolabeled trastuzumab binding to HER2.

The positron-emitting isotope ${ }^{64} \mathrm{Cu}$ is regularly available from Washington University, St. Louis, and we have extensive experience labeling antibodies with radiometals via the chelating agent DOTA (12). Although its half-life $(12.8 \mathrm{~h})$ is short relative to the blood clearance of trastuzumab, ${ }^{64} \mathrm{Cu}$ has potential advantages over ${ }^{89} \mathrm{Zr}$ in terms of radiation safety and patient radiation dose. The critical issue, addressed in this study, is whether tumor uptake of ${ }^{64} \mathrm{Cu}$-DOTA-trastuzumab is sufficiently rapid to support tumor imaging and quantification within the 48-h time window afforded by ${ }^{64} \mathrm{Cu}$. On the basis of our previous clinical study with ${ }^{111} \mathrm{In}-2-$ (p-isothiocyanatobenzyl)-6-methyldiethylenetriamino- $N, N, N^{\prime}, N^{\prime \prime}$, $N^{\prime \prime}$-pentaacetic acid (MX-DTPA)-trastuzumab (9) and promising results in athymic mice bearing HER2-expressing human breast adenocarcinoma xenografts (13), we have obtained an investigational new drug (IND) application for ${ }^{64} \mathrm{Cu}$-DOTA-trastuzumab.

The primary objective of this pilot study was to evaluate the feasibility and potential utility of CT-supplemented PET scanning of ${ }^{64} \mathrm{Cu}$-DOTA-trastuzumab $\left({ }^{64} \mathrm{Cu}\right.$-DOTA-trastuzumab PET/CT) for lesion detection and uptake measurement in HER2-positive metastatic breast cancer. Similar to other antibodies (14), liver uptake of intravenously administered trastuzumab is strongly dependent on antibody protein load. Thus, we sought to identify a trastuzumab dose that minimizes liver uptake of ${ }^{64} \mathrm{Cu}$-DOTA-trastuzumab. Additional goals were to compare ${ }^{64} \mathrm{Cu}$-DOTA-trastuzumab with the standard PET radiotracer, ${ }^{18} \mathrm{~F}-\mathrm{FDG}$, and to confirm the safety of the ${ }^{64} \mathrm{Cu}$-DOTA-trastuzumab $\mathrm{PET} / \mathrm{CT}$ procedure.

\section{MATERIALS AND METHODS}

\section{Patient Selection}

Women with metastatic HER2-positive breast cancer who had not received HER2-directed therapy for 4 mo or more were considered for study participation after undergoing a staging workup that included echocardiogram, bone scanning, and whole-body ${ }^{18} \mathrm{~F}-\mathrm{FDG}$ PET/CT. All candidates underwent biopsy of a metastatic lesion within $28 \mathrm{~d}$ before the ${ }^{64} \mathrm{Cu}$-DOTA-trastuzumab procedure to confirm recurrent, HER2-positive disease by immunohistochemical staining or fluorescence in situ hybridization. Assessable disease outside the primary breast site, ipsilateral axillary region, and biopsy site was also required. The study protocol was approved by the City of Hope Institutional Review Board and Radiation Safety Committee, and an IND was accepted by the Food and Drug Administration. All patients signed a written informed consent form.

\section{${ }^{64} \mathrm{Cu}$-DOTA-Trastuzumab Preparation}

Trastuzumab is a recombinant humanized antibody that binds with high affinity to the extracellular domain of the HER2 protein. Radiolabeled trastuzumab was prepared according to procedures defined in IND \#109971. The antibody (Herceptin, purchased from Genentech) was conjugated with the active ester of DOTA (Macrocyclics) under current good manufacturing-compliant conditions. ${ }^{64} \mathrm{Cu}$ (half-life, $12.8 \mathrm{~h}$; 0.18 positrons/decay) was provided by the Mallinckrodt Institute of Radiology, Washington University School of Medicine. DOTA-conjugated antibody was incubated with ${ }^{64} \mathrm{Cu}$ for $45 \mathrm{~min}$ at $43{ }^{\circ} \mathrm{C}$, chased with $1 \mathrm{mM}$ diethylenetriamine pentaacetic acid (DTPA), and purified on a size-exclusion, preparative column (Superdex-200; GE Healthcare Life Sciences). Radiolabeling efficiency was more than 93\%. Appropriate fractions were pooled, filtered, and formulated with $1 \%$ human serum albumin for patient administration. The ${ }^{64} \mathrm{Cu}$-DOTA-trastuzumab preparations were sterile, with endotoxin levels less than $0.05 \mathrm{EU} / \mathrm{mL}$ and immunoreactivity greater than $86 \%$. The DOTA-trastuzumab protein dose per ${ }^{64} \mathrm{Cu}$-DOTA-trastuzumab injection was approximately $5 \mathrm{mg}$.

\section{Administration of Trastuzumab and ${ }^{64} \mathrm{Cu}$-DOTA-Trastuzumab}

Patients were closely monitored for acute adverse reactions during trastuzumab administrations. ${ }^{64} \mathrm{Cu}$-DOTA-trastuzumab (364-512 MBq; mean, $450 \mathrm{MBq}$ ) was infused intravenously in $25 \mathrm{~mL}$ of saline over $10 \mathrm{~min}$. Dijkers et al. found that, compared with $10 \mathrm{mg}, 50 \mathrm{mg}$ of trastuzumab substantially reduced blood clearance and liver uptake of ${ }^{89} \mathrm{Zr}$-trastuzumab in trastuzumab-naïve patients $(10)$. To match the trastuzumab dose found effective in that study, patients receiving nonradiolabeled trastuzumab were infused intravenously with the antibody ( $45 \mathrm{mg}$ in $50 \mathrm{~mL}$ of saline given over $15 \mathrm{~min}$ ) immediately before radioactive injection.

The first 4 patients in our study were randomly assigned to receive trastuzumab doses of 5 or $50 \mathrm{mg}$. When ${ }^{64} \mathrm{Cu}$-DOTA-trastuzumab PET/CT of those patients confirmed the findings of Dijkers et al., we adopted the 50-mg dose for the remainder of the study.

\section{PET/CT Imaging}

Imaging was performed with a Discovery STe 16 PET/CT scanner (GE Healthcare) operated in 3-dimensional mode (septa retracted). The PET axial field of view is $15.4 \mathrm{~cm}$ (image slice thickness, $3.3 \mathrm{~mm}$ ). PET images were reconstructed using an iterative, ordered-subsets expectation maximization algorithm with gaussian postsmoothing and standard corrections for nonuniform detector sensitivity, scanner dead time, and both random and scattered coincidence events. Correction for photon attenuation was based on coregistered CT scans acquired during the same examination. The measured spatial resolution of the PET images was approximately $9 \mathrm{~mm}$ (full width at half maximum).

Patients underwent a standard ${ }^{18} \mathrm{~F}-\mathrm{FDG}$ PET/CT examination $13 \mathrm{~d}$ or fewer before the ${ }^{64} \mathrm{Cu}$-DOTA-trastuzumab procedure. Patients fasted 6 $\mathrm{h}$ or more before injection of ${ }^{18} \mathrm{~F}$-FDG. Serum glucose concentration measured at time of examination was high $(184 \mathrm{mg} / \mathrm{dL})$ for one patient and normal $(<120 \mathrm{mg} / \mathrm{dL})$ for the others.

Injected ${ }^{64} \mathrm{Cu}$ activity was limited to $555 \mathrm{MBq}(15 \mathrm{mCi})$, based on radiation dose estimates calculated from the pharmacokinetics of ${ }^{111} \mathrm{In}$ MxDTPA-trastuzumab (9). One hour was chosen as a reasonable limit for PET scan duration. Within those constraints, disease location as judged from the preceding ${ }^{18} \mathrm{~F}$-FDG PET/CT examination was used in choosing the axial coverage for the ${ }^{64} \mathrm{Cu}$-DOTA-trastuzumab PET/CT scans. The first (day 1) ${ }^{64} \mathrm{Cu}$ scan was performed $21-25 \mathrm{~h}$ after injection to allow radiolabeled antibody accumulation in tumor. A second (day 2) scan was obtained 47-49 h after injection. Day 1 scans comprised 3 or 4 (39 or $51 \mathrm{~cm}$ axial extent, 20 or 15 min per bed position) and day 2 scans comprised 1 or 2 (15 or $27 \mathrm{~cm}$ axial extent, 60 or $30 \mathrm{~min}$ per bed position) contiguous bed positions, respectively, depending on patient body thickness. Signal-to-noise characteristics of the ${ }^{64} \mathrm{Cu}$-DOTA-trastuzumab images approximated those of the ${ }^{18} \mathrm{~F}$ FDG scans (Fig. 1).

\section{Image Analysis}

$\mathrm{PET} / \mathrm{CT}$ examinations were interpreted by a radiologist board-certified in nuclear medicine. Tumor detection sensitivity and uptake analyses were limited to lesions identifiable on CT; lesions visualized relative to adjacent tissue on PET were considered PET-positive. PET-positive lesions were disregarded if $\mathrm{CT}$ was judged inconclusive. PET-positive findings with ${ }^{64} \mathrm{Cu}$-DOTA-trastuzumab but not ${ }^{18} \mathrm{~F}$-FDG, and having no correlated CT lesion, were scored as false-positives. Because of possible ${ }^{18} \mathrm{~F}-\mathrm{FDG}$ or nonspecific antibody uptake secondary to biopsy, biopsied tumor sites were not included in the analysis. A detailed description of the lesion detection analysis is given in the supplemental material (available only online at http://jnm.snmjournals.org). 


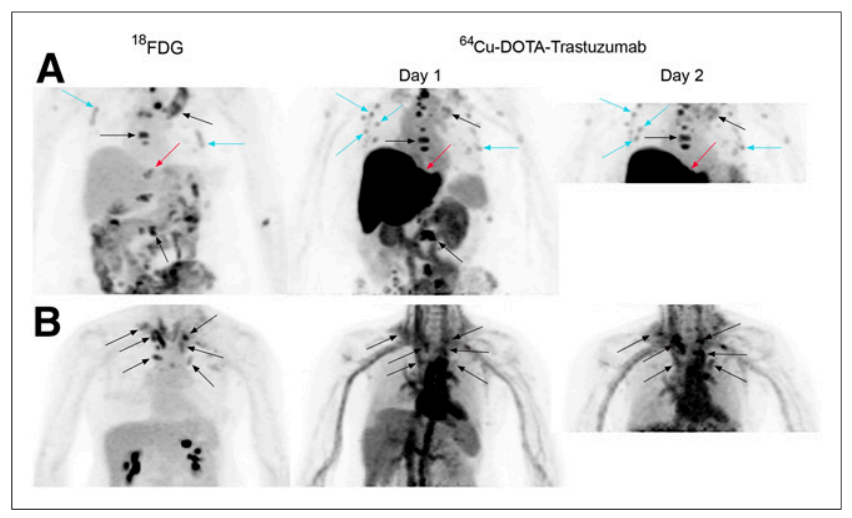

FIGURE 1. Visualization of HER2-positive metastatic breast cancer by PET of ${ }^{64} \mathrm{Cu}$-DOTA-trastuzumab. Images are maximum-intensity projections with upper intensity thresholds corresponding to SUV $=10 \mathrm{~g} / \mathrm{mL}$. (A) PET scans of ${ }^{18} \mathrm{~F}-\mathrm{FDG},{ }^{64} \mathrm{Cu}$-DOTA-trastuzumab $23 \mathrm{~h}$ after injection (day 1), and ${ }^{64} \mathrm{Cu}$-DOTA-trastuzumab $48 \mathrm{~h}$ after injection (day 2 ) in a patient (patient A) who received trastuzumab dose of $5 \mathrm{mg}$. Black arrows point out several of many corresponding CT-positive bone lesions seen both with ${ }^{18} \mathrm{~F}-\mathrm{FDG}$ and with ${ }^{64} \mathrm{Cu}$-DOTA-trastuzumab, whereas turquoise arrows denote a few of many instances of ${ }^{64} \mathrm{Cu}$-DOTA-trastuzu$\mathrm{mab}$, or ${ }^{64} \mathrm{Cu}$-DOTA-trastuzumab and ${ }^{18} \mathrm{~F}-\mathrm{FDG}$, focal uptake in rib regions too small to be evaluated on associated CT. Red arrows indicate intrahepatic lesion seen with ${ }^{18} \mathrm{~F}-\mathrm{FDG}$ but obscured by intense liver uptake in ${ }^{64} \mathrm{Cu}$-DOTA-trastuzumab scans. (B) ${ }^{18} \mathrm{~F}-\mathrm{FDG},{ }^{64} \mathrm{Cu}$-DOTAtrastuzumab $24 \mathrm{~h}$ after injection, and ${ }^{64} \mathrm{Cu}$-DOTA-trastuzumab $48 \mathrm{~h}$ after injection scans of patient (patient B) given trastuzumab dose of $50 \mathrm{mg}$. Arrows denote lymph nodes visualized in both ${ }^{18} \mathrm{~F}-\mathrm{FDG}$ and ${ }^{64} \mathrm{Cu}-$ DOTA-trastuzumab scans. Liver uptake of ${ }^{64} \mathrm{Cu}$ was much lower for patient $B$ than for patient $A$ (compare day 1 images).

Radiolabel uptake in as many as 10 of the most prominent lesions per patient, as well as selected nontumor tissues and organs, was measured in terms of standardized uptake value $(\mathrm{SUV}=$ tissue activity per $\mathrm{cm}^{3} \times$ body weight $[\mathrm{g}] /$ injected activity decay-corrected to time of scan). Tumor uptake was parameterized as single-voxel maximum SUV ( $\mathrm{SUV}_{\max }$ ) and background-adaptive whole-tumor average SUV (15). We found whole-tumor SUV to be closely and linearly correlated with $\mathrm{SUV}_{\max }\left(r^{2} \geq 0.97, P<0.001\right)$. Therefore, tumor uptake results are presented only in terms of $\mathrm{SUV}_{\max }$.

Uptake analysis for blood, liver, spleen, kidney, and heart wall consisted of averaging the mean SUVs of circular or elliptic regions of interest of fixed size placed well within the tissue's or organ's PET image boundaries on 3 contiguous image slices. Blood measurements were obtained from PET images of the cardiac ventricles; the heart wall was visualized as a region of relatively low uptake adjacent to the ventricles.

\section{Pharmacokinetic Analysis and Radiation Dose Estimates}

${ }^{64} \mathrm{Cu}$ activity concentration was measured in peripheral venous samples acquired $0-1,23-24$, and $47-48 \mathrm{~h}$ after injection from patients who received a 50-mg trastuzumab dose. Radiation dose estimates for these patients were obtained by combining blood activity and organ uptake measurements from the current study with blood and organ time-activity measurements $(0-168 \mathrm{~h})$ from our previous clinical study with ${ }^{111}$ In-MxDTPA-trastuzumab (9). Details of the radiation dose calculations are given in the supplemental material.

\section{Human Anti-Trastuzumab Antibody Response}

Serum samples obtained just before trastuzumab $/{ }^{64} \mathrm{Cu}$-DOTAtrastuzumab infusion and 1, 3, and 6 mo later, when possible, were evaluated for immune responses using a size-exclusion high-performance liquid chromatography (HPLC) shift assay. Samples $(125 \mu \mathrm{L})$ were incubated with radiolabeled DOTA-trastuzumab $\left({ }^{111} \mathrm{In}, 0.33[9 \mu \mathrm{Ci}]\right.$ $\mathrm{MBq} / \mu \mathrm{g}, 3.7 \mathrm{kBq}[0.1 \mu \mathrm{Ci}])$ and then run on a Superose- 6 size-exclusion column (GE Healthcare Life Sciences) at $0.4 \mathrm{~mL} / \mathrm{min}$ in phosphatebuffered saline $/ 0.05 \% \mathrm{NaN}_{3}$. A change in the elution pattern of the radiolabeled trastuzumab consistent with higher molecular weight was considered positive for an anti-antibody response.

\section{Statistical Analysis}

Statistical analysis was performed using R (version 2.12.1, The R Foundation of Statistical Computing). Lesion detection sensitivities were compared by a 2-sided Fisher exact test. Comparison of tumor uptake between trastuzumab doses and among lesion sites used ANOVA to evaluate both dose/lesion site and patient effects, with Holm's method to adjust for multiple comparisons. Linear regression analysis was used to demonstrate correlation between whole-tumor SUV and $\mathrm{SUV}_{\text {max }}$, as well as lack of correlation between ${ }^{18} \mathrm{~F}-\mathrm{FDG}$ and ${ }^{64} \mathrm{Cu}$-DOTA-trastuzumab. The effect of trastuzumab dose on organ uptake was evaluated by Wilcoxon rank-sum test. $P$ values of less than 0.05 were considered statistically significant.

\section{RESULTS}

\section{Patient Characteristics}

Eight of 10 women considered for study participation met the eligibility criteria. Biopsies of 2 patients previously treated for early stage HER2-positive breast cancer showed recurrent disease to be HER2-negative. Participating patients are characterized in Table 1.

TABLE 1

Patient Demographics and Clinical Characteristics*

\begin{tabular}{|c|c|c|c|}
\hline \multirow[b]{2}{*}{ Characteristic } & \multicolumn{2}{|c|}{$\begin{array}{l}\text { Trastuzumab } \\
\text { protein dose } \\
\quad(\mathrm{mg})\end{array}$} & \multirow[b]{2}{*}{ All patients } \\
\hline & 5 & 50 & \\
\hline No. of patients & 2 & 6 & 8 \\
\hline \multicolumn{4}{|l|}{ Age $(y)$} \\
\hline Median & 60 & 54 & 56 \\
\hline Range & $44-75$ & $39-69$ & $39-75$ \\
\hline \multicolumn{4}{|l|}{ Prior anti-HER2 therapy } \\
\hline None & & 1 & 1 \\
\hline Adjuvant trastuzumab & 1 & 2 & $3(14,18,18)^{\dagger}$ \\
\hline $\begin{array}{l}\text { Trastuzumab for } \\
\text { metastasis }\end{array}$ & 1 & 3 & $4(4,6,14,18)^{\dagger}$ \\
\hline \multicolumn{4}{|l|}{$\begin{array}{l}\text { Hormone receptor and } \\
\text { HER2 status of } \\
\text { recurrent disease }\end{array}$} \\
\hline ER or PR positive & 1 & 3 & 4 \\
\hline ER and PR negative & 1 & 3 & 4 \\
\hline \multicolumn{4}{|l|}{ HER2 } \\
\hline $\mathrm{IHC} 3+$ & 2 & 5 & 7 \\
\hline IHC2+/FISH positive & & 1 & 1 \\
\hline \multicolumn{4}{|l|}{$\begin{array}{l}\text { Sites of metastatic } \\
\text { disease }\end{array}$} \\
\hline Bone & 2 & 4 & 6 \\
\hline Lymph nodes & 2 & 5 & 7 \\
\hline Liver & 2 & 2 & 4 \\
\hline Lung & 1 & 1 & 2 \\
\hline Pleural effusion & & 1 & 1 \\
\hline Breast & & 2 & 2 \\
\hline
\end{tabular}

*Entries are numbers of patients unless otherwise indicated.

${ }^{\dagger}$ Months since last anti-HER2 therapy administration.

$\mathrm{ER}=$ estrogen receptor; $\mathrm{PR}=$ progesterone receptor; $\mathrm{IHC}=$ immunohistochemistry; FISH = fluorescence in situ hybridization. 


\section{Lesion Detection Sensitivity of ${ }^{64} \mathrm{Cu}$-DOTA-Trastuzumab PET/CT}

Figure 1 illustrates ${ }^{64} \mathrm{Cu}$-DOTA-trastuzumab image quality and tumor visualization, compared with ${ }^{18} \mathrm{~F}$-FDG. Tumor-to-nontumor contrast for ${ }^{64} \mathrm{Cu}$-DOTA-trastuzumab was generally high (Fig. 1, patient A). Exceptions occurred for lymph nodes in the cervical, clavicular, and mediastinal regions due to high blood-pool activity (Fig. 1, patient B) and in the liver for the 5-mg trastuzumab dose (Fig. 1, patient A). Visualization of lymph nodes in regions of high blood activity improved between day 1 and day 2 but changed little for other lesion sites between the 2 scans (Fig. 2).

Lesion detection statistics are summarized in Table 2. Overall detection sensitivity with ${ }^{18}$ F-FDG PET/CT (93\%) was consistent with general experience in metastatic breast cancer (16). All 8 patients had CT-positive lesions that were detected with ${ }^{64} \mathrm{Cu}$-DOTAtrastuzumab PET. There were no statistically significant differences in ${ }^{64} \mathrm{Cu}$ lesion detection sensitivity between 5- and 50-mg trastuzumab doses (data not shown). On day $1,{ }^{64} \mathrm{Cu}$ detection sensitivity was lower for lymph nodes than for bone lesions. Overall, detection sensitivity for ${ }^{64} \mathrm{Cu}$-DOTA-trastuzumab on day 1 was lower than for ${ }^{18} \mathrm{~F}$-FDG, with the difference being due primarily to the low sensitivity of lymph nodes in regions of high blood activity. There were 7 instances in which a CT-positive lesion was detected with ${ }^{18} \mathrm{~F}$-FDG but not ${ }^{64} \mathrm{Cu}$-DOTA-trastuzumab on either day 1 or day 2. In 6 instances ( 3 bone, 2 liver, and 1 node), a CT-positive lesion was detected with ${ }^{64} \mathrm{Cu}$-DOTA-trastuzumab but not with ${ }^{18}$ F-FDG. ${ }^{18} \mathrm{~F}$ FDG false-negative bone and liver lesions are illustrated in Figures 2 and 3B, respectively.

There was only 1 instance of a false-positive ${ }^{64} \mathrm{Cu}$-DOTA-trastuzumab lesion, which occurred in the colon and may have been associated with diverticulitis. In 1 patient with numerous bone metastases, ${ }^{64} \mathrm{Cu}-$ DOTA-trastuzumab, or both ${ }^{64} \mathrm{Cu}$-DOTA-trastuzumab and ${ }^{18} \mathrm{~F}$ FDG, produced hot spots in rib regions too small to be assessed on associated CT (Fig. 1, patient A).

\section{Effects of Trastuzumab Protein Dose}

Blood clearance was slowed, and liver uptake of ${ }^{64} \mathrm{Cu}$-DOTAtrastuzumab was markedly decreased in patients preinfused with trastuzumab (45 mg) (Supplemental Fig. 1). However, with only 2 patients at the lower protein dose, SUV differences between the 50- and 5-mg trastuzumab doses were not statistically significant $(P=0.10$ and 0.05 , respectively, on days 1 and 2 for blood; $P=$ 0.10 and 0.07 , respectively, on days 1 and 2 for liver). Trastuzumab predosing dramatically improved visualization of hepatic metastases (Fig. 3) and had little effect on ${ }^{64} \mathrm{Cu}$-DOTA-trastuzumab uptake in the heart wall (Supplemental Fig. 1), kidney, or spleen (data not shown).

No statistically significant difference in tumor uptake of ${ }^{64} \mathrm{Cu}$ DOTA-trastuzumab was observed between the 2 trastuzumab doses. Tumor $\mathrm{SUV}_{\max }$ was generally higher for the 5- than for the 50-mg dose on day 1 (mean $\pm \mathrm{SD}, 11.3 \pm 5.9$, compared with $6.7 \pm 2.4$, $P=0.01$ ) but trended in the other direction on day 2 (mean $\pm \mathrm{SD}$, $5.9 \pm 3.7$, compared with $9.6 \pm 5.9, P=0.11)$. When ANOVA included both a patient effect (i.e., accounted for varying numbers of lesions among different patients) and a dose effect, there was no significant trastuzumab dose effect on either day.

\section{Heterogeneity of ${ }^{64} \mathrm{Cu}$-DOTA-Trastuzumab Uptake in Tumors}

Uptake varied widely both among and within patients (Fig. 4). For the data included in Figure 4, mean $\mathrm{SUV}_{\max }$ ranged from 5.5 to $15.0 \mathrm{~g} / \mathrm{mL}$ among the 8 patients. Within patients, $\mathrm{SUV}_{\max }$

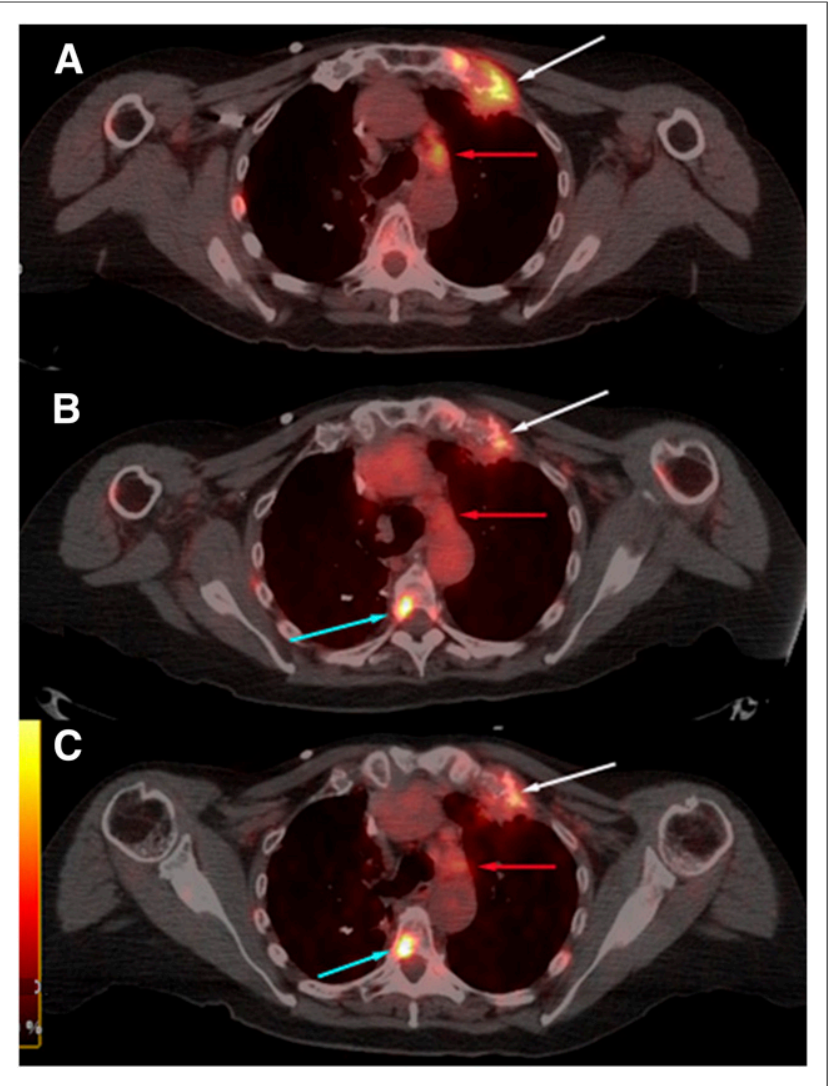

FIGURE 2. Visualization of bone and nodal metastases. Shown are transaxial PET/CT fusion images of ${ }^{18} \mathrm{~F}-\mathrm{FDG}(\mathrm{A}),{ }^{64} \mathrm{Cu}$-DOTA-trastuzumab $23 \mathrm{~h}$ after injection (B), and ${ }^{64} \mathrm{Cu}$-DOTA-trastuzumab $48 \mathrm{~h}$ after injection (C) from patient given $50 \mathrm{mg}$ of trastuzumab. Upper intensity thresholds (white color) correspond to SUV $=10 \mathrm{~g} / \mathrm{mL}$. Lesion-to-nonlesion contrast improved modestly between day 1 and day 2 after ${ }^{64} \mathrm{Cu}-$ DOTA-trastuzumab injection. Lesion growing out of left first rib (white arrows) is well visualized on day $1{ }^{64} \mathrm{Cu}$ scan and little changed on day 2 . Spinal metastasis (turquoise arrows) not seen with ${ }^{18} \mathrm{~F}-\mathrm{FDG}$ is well visualized on both day 1 and day $2{ }^{64} \mathrm{Cu}$ scans. On the other hand, nodal metastasis (red arrows) seen with ${ }^{18} \mathrm{~F}-\mathrm{FDG}$ is visualized only on day 2 with ${ }^{64} \mathrm{Cu}$-DOTA-trastuzumab.

varied between 2- and 5-fold in 7 patients and 22-fold in 1 patient. The variability was, in part, associated with lesion site (Fig. 5).

\section{Tumor Uptake Compared Between ${ }^{64} \mathrm{Cu}$-DOTA-Trastuzumab and ${ }^{18} \mathrm{~F}-\mathrm{FDG}$}

Uptake of ${ }^{64} \mathrm{Cu}$-DOTA-trastuzumab and ${ }^{18} \mathrm{~F}-\mathrm{FDG}$ was comparable when averaged over all lesions. For combined 5- and 50-mg trastuzumab doses, $\mathrm{SUV}_{\max }$ results (mean, median, range) were: ${ }^{18} \mathrm{~F}-\mathrm{FDG}(9.7,9.3$, 3.3-25.4, $n=56)$; ${ }^{64} \mathrm{C}$-DOTA-trastuzumab day 1 (8.1, 7.0, 3.0-22.5, $n=48) ;{ }^{64}$ C-DOTA-trastuzumab day 2 (9.0, 7.5, 0.9-28.9, $\left.n=38\right)$.

ANOVA including both lesion site and patient effects indicated significant lesion site effects for both ${ }^{64} \mathrm{Cu}$-DOTA-trastuzumab and ${ }^{18}$ F-FDG (Fig. 5). Pairwise comparisons between sites showed ${ }^{18} \mathrm{~F}-\mathrm{FDG}$ uptake in liver metastases to be less than in bone metastases $(P<0.01)$, whereas ${ }^{64} \mathrm{Cu}$-DOTA-trastuzumab uptake on day 2 was higher in liver metastases than in bone metastases $(P<0.02)$.

Same-lesion $\mathrm{SUV}_{\max }$ for ${ }^{64} \mathrm{Cu}$-DOTA-trastuzumab and ${ }^{18} \mathrm{~F}$-FDG was uncorrelated $(P \geq 0.4$; correlation coefficients $=-0.1)$. $\mathrm{SUV}_{\max }$ ratios $\left({ }^{64} \mathrm{Cu}\right.$-DOTA-trastuzumab to ${ }^{18} \mathrm{~F}$-FDG) varied from 0.2 to 4.3 (Supplemental Fig. 2). 
TABLE 2

Detection of CT-Positive Lesions with PET

\begin{tabular}{|c|c|c|c|}
\hline \multirow[b]{2}{*}{ Lesion site } & \multirow[b]{2}{*}{${ }^{18} \mathrm{~F}-\mathrm{FDG}^{*}$} & \multicolumn{2}{|c|}{${ }^{64} \mathrm{Cu}$-DOTA-trastuzumab ${ }^{\dagger}$} \\
\hline & & Day 1 & Day 2 \\
\hline All & 83 of $89(93 \%)$ & 61 of $79(77 \%)^{\ddagger}$ & 54 of $61(89 \%)$ \\
\hline Bone & 35 of $38(92 \%)$ & 33 of $36(92 \%)$ & 19 of $20(95 \%)$ \\
\hline Lymph nodes & 30 of $31(97 \%)$ & 20 of $31(65 \%)^{\S, \|}$ & 19 of $23(88 \%)$ \\
\hline Liver & 8 of $10(80 \%)$ & 1 of $3(33 \%)$ & 8 of $8(100 \%)$ \\
\hline Lung & 5 of $5(100 \%)$ & 4 of $4(100 \%)$ & 4 of $5(80 \%)$ \\
\hline Pleural effusion & 2 of $2(100 \%)$ & 0 of $2(0 \%)$ & 1 of $2(50 \%)$ \\
\hline Breast & 3 of $3(100 \%)$ & 3 of $3(100 \%)$ & 3 of $3(100 \%)$ \\
\hline \multicolumn{4}{|c|}{$\begin{array}{l}{ }^{*} \text { Lesions evaluated for }{ }^{18} \mathrm{~F}-\mathrm{FDG} \text { were all included in and evaluated for either or both of the }{ }^{64} \mathrm{Cu} \text {-DOTA-trastuzumab scans. } \\
{ }^{\dagger} \text { Combined data for } 5-\text { and } 50-\mathrm{mg} \text { trastuzumab doses. } \\
{ }^{\ddagger} P<0.01 \text { relative to }{ }^{18} \mathrm{~F}-\mathrm{FDG} \text {, all sites. } \\
{ }^{\$} P<0.01 \text { relative to }{ }^{18} \mathrm{~F}-\mathrm{FDG} \text {, lymph nodes. } \\
\|_{P}<0.05 \text { relative to }{ }^{64} \mathrm{Cu} \text {-DOTA-trastuzumab day } 1 \text {, bone. }\end{array}$} \\
\hline
\end{tabular}

\section{Patient Safety}

Trastuzumab infusion and ${ }^{64} \mathrm{Cu}$-DOTA-trastuzumab PET/CT were well tolerated, with no unanticipated toxicity or adverse side effects observed. Anti-trastuzumab antibody response assays were negative for 6 patients. Minor increases in higher-molecular-weight complexes were observed in the HPLC shift assays of 2 patients at baseline and $6 \mathrm{mo}$ or baseline, 3 , and $6 \mathrm{mo}$ after their ${ }^{64} \mathrm{Cu}$-DOTAtrastuzumab PET/CT procedures. Estimated radiation doses (Table 3 ) were well within the range of those for established radionuclear imaging procedures.

\section{DISCUSSION}

Tumor HER2 status and trastuzumab exposure history were more clearly prescribed in the current investigation than in prior imaging studies with trastuzumab (8-10). All patients had biopsy confirmation of HER 2 positivity at the time of study, and none had received anti-HER2 therapy for at least 4 mo before imaging.

We have clearly shown that, despite the relatively short half-life of the radiolabel, ${ }^{64} \mathrm{Cu}$-DOTA-trastuzumab PET/CT can effectively detect and quantify tumor uptake in patients with known HER2positive disease. Other than the brain, all anatomic sites common to metastatic breast cancer were included in the patient cohort. Lesions were visualized in all 8 patients examined and were seen in bone, lymph nodes, liver, lung, pleural effusions, and breast. Detection sensitivity was $77 \%$ on day 1 and $89 \%$ on day 2 (Table 2). Tumor uptake was substantial by $24 \mathrm{~h}$ and, on average, increased modestly between 24 and $48 \mathrm{~h}$. Detection of lymph nodes in the neck, upper thorax, and mediastinum is difficult at $24 \mathrm{~h}$ because of high blood background but improves by $48 \mathrm{~h}$ (Fig. 2). The chief limitation of ${ }^{64} \mathrm{Cu}$-DOTA-trastuzumab PET/CT is that, because of the 13 -h half-life of ${ }^{64} \mathrm{Cu}$, it does not provide whole-body coverage with acceptable signal-to-noise ratio and scan duration. Nonetheless, as demonstrated here, ${ }^{64} \mathrm{Cu}$-DOTAtrastuzumab PET can be used effectively in disseminated, HER2positive breast cancer when disease location is defined in advance by ${ }^{18}$ F-FDG PET or CT.

A second major objective was to establish a trastuzumab protein load that minimizes liver uptake without inhibiting tumor uptake of ${ }^{64} \mathrm{Cu}$-DOTA-trastuzumab. We observed that adding $45 \mathrm{mg}$ of trastuzumab to the $5 \mathrm{mg}$ of DOTA-trastuzumab delivered with the radioactive injection approximately doubled blood SUV and re- duced liver uptake by $75 \%-80 \%$ on days 1 and 2 after radiotracer injection (Supplemental Fig. 1). These observations are quantitatively similar to those reported by Dijkers et al. for ${ }^{89} \mathrm{Zr}$-trastuzumab given with trastuzumab loads of 10 and $50 \mathrm{mg}(10)$.

Comparison with our ${ }^{111} \mathrm{In}-\mathrm{MxDTPA}$-trastuzumab study (trastuzumab load 4-8 mg/kg) suggests that increasing beyond $50 \mathrm{mg}$ of trastuzumab dose would not yield further improvement in the pharmacokinetics or biodistribution of ${ }^{64} \mathrm{Cu}$-DOTA-trastuzumab. We observed no statistically significant difference in tumor uptake between 5- and 50-mg doses in this small study. However, other investigations have demonstrated that a significant fraction of tumor binding sites can be occupied at antibody loading doses $<<4-8 \mathrm{mg} / \mathrm{kg}$ (17). Further-

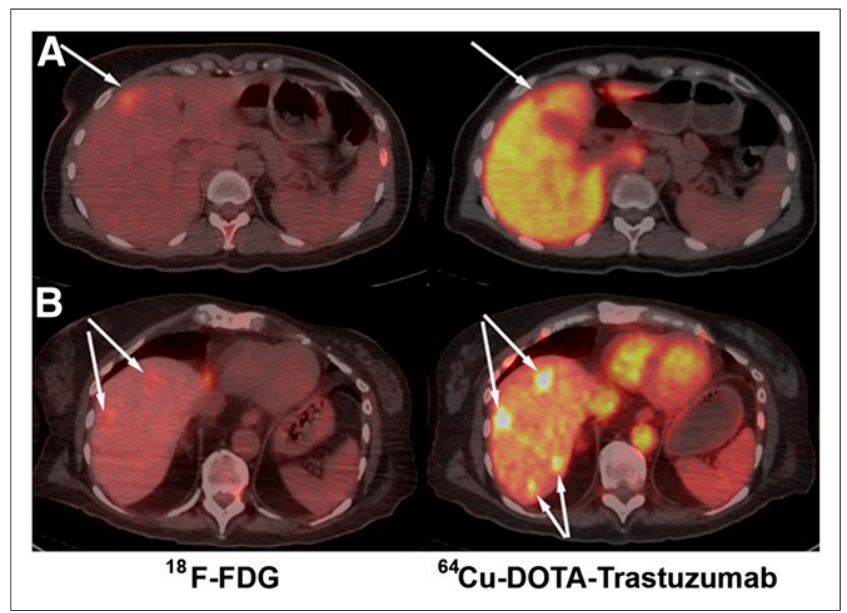

FIGURE 3. Visualization of hepatic metastases. (A) Fused transaxial PET/CT images of ${ }^{18} \mathrm{~F}$-FDG and ${ }^{64} \mathrm{Cu}$-DOTA-trastuzumab $21 \mathrm{~h}$ after injection for patient (patient A) given $5 \mathrm{mg}$ of trastuzumab. Upper intensity thresholds (white color) correspond to SUV $=10 \mathrm{~g} / \mathrm{mL}$ for ${ }^{18} \mathrm{~F}-\mathrm{FDG}$ and $40 \mathrm{~g} / \mathrm{mL}$ for ${ }^{64} \mathrm{Cu}$-DOTA-trastuzumab. (B) Fused transaxial PET/CT images of ${ }^{18} \mathrm{~F}-\mathrm{FDG}$ and ${ }^{64} \mathrm{Cu}$-DOTA-trastuzumab $47 \mathrm{~h}$ after injection for patient (patient B) given $50 \mathrm{mg}$ of trastuzumab. Upper intensity thresholds correspond to SUV $=10 \mathrm{~g} / \mathrm{mL}$. Arrows indicate detected lesions. For patient $A$, anterior hepatic lesion is visualized as cold spot on ${ }^{64} \mathrm{Cu}$-DOTA-trastuzumab scan. Liver SUV was $27.6 \mathrm{~g} / \mathrm{mL}$ in patient $A$, compared with $5.5 \mathrm{~g} / \mathrm{mL}$ in patient $B$, for whom hepatic lesions are dramatically visualized as hot spots. For patient $\mathrm{B}, 2$ lesions not seen with ${ }^{18} \mathrm{~F}-\mathrm{FDG}$ are detected with ${ }^{64} \mathrm{Cu}$-DOTA-trastuzumab. 


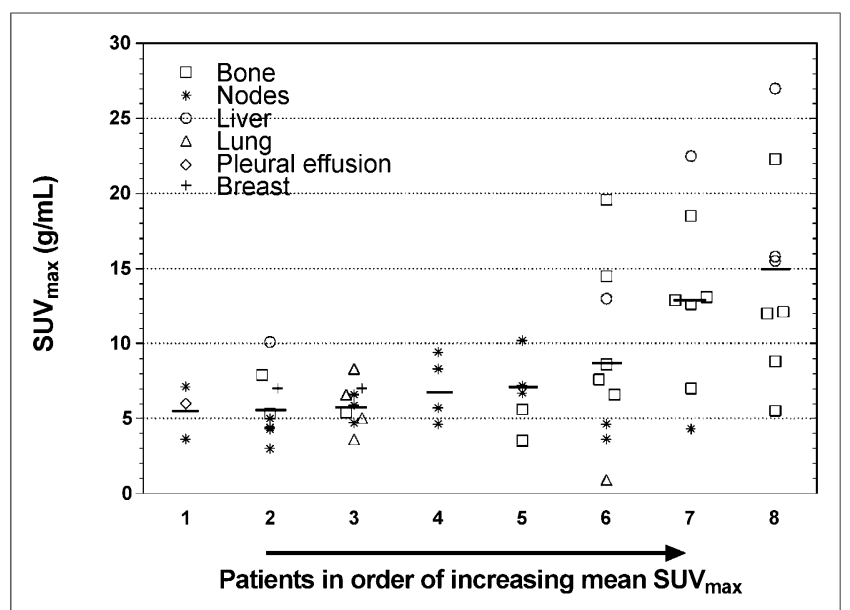

FIGURE 4. Inter- and intrapatient heterogeneity of ${ }^{64} \mathrm{Cu}$-DOTA-trastuzumab tumor uptake. Short horizontal lines indicate intrapatient mean SUV $_{\max }$. Data are from day $1(n=49)$ or day $2(n=7)$ for lesions not included in day 1 scan. Six of day 2 lesions are for patient 8 , for whom data from 2 of 3 scanned bed positions on day 1 were lost due to scanner malfunction. Other day 2 lesion is for patient 6 . Patients 6 and 7 received trastuzumab doses of $5 \mathrm{mg}$; others received $50 \mathrm{mg}$.

more, the dissociation constant for ${ }^{111}$ In-DTPA-trastuzumab-HER2 binding is approximately $10 \mathrm{nM}(18)$, a concentration that very likely would be exceeded in tumors at a trastuzumab load of $4-8 \mathrm{mg} / \mathrm{kg}$. This suggests that HER2 saturation may have contributed to the relatively low tumor detection sensitivity in our ${ }^{111} \mathrm{In}$-MxDTPA-trastuzumab study (4 lesions visualized in 3 of 7 patients with known lesions).

Heterogeneity of tumor HER2 expression within and among patients is poorly understood (5-7) and may be elucidated by imaging studies with radiolabeled trastuzumab. The high degree of tumor positivity observed in the current study suggests that most lesions in HER2-positive patients have HER2 expression adequate to render them detectable with ${ }^{64} \mathrm{Cu}$-DOTA-trastuzumab PET/CT. On the other hand, tumor uptake was also highly variable among and within patients (Fig. 4). That heterogeneity suggests a potential role for ${ }^{64} \mathrm{Cu}$-DOTA-trastuzumab PET/CT in the selection of patients for trastuzumab-based therapy.

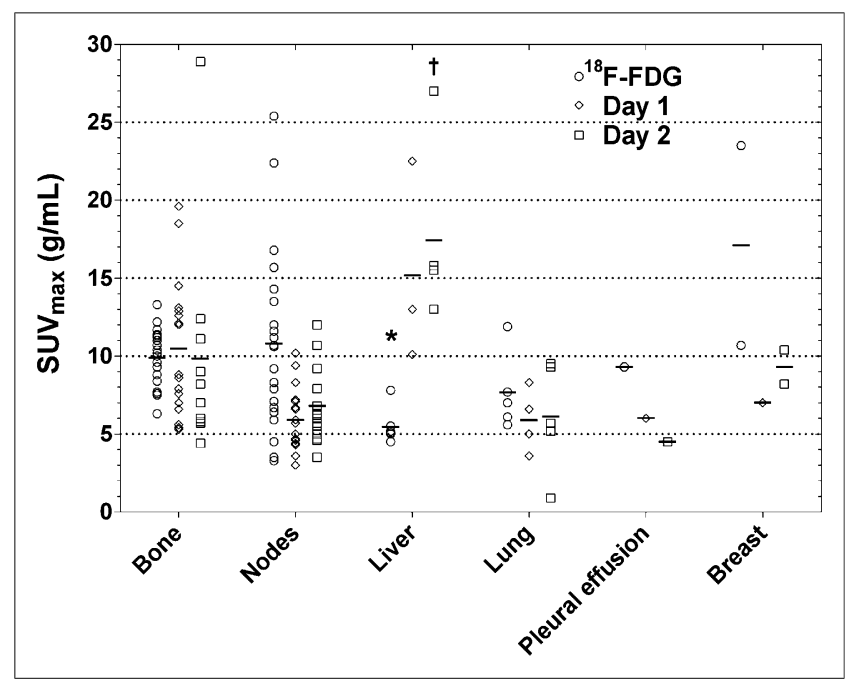

FIGURE 5. Tumor uptake of ${ }^{18} \mathrm{~F}-\mathrm{FDG}$ and ${ }^{64} \mathrm{Cu}$-DOTA-trastuzumab (day 1 and day 2) vs. lesion site. Short horizontal lines indicate intrasite averages. ${ }^{*}<{ }^{18} \mathrm{~F}-\mathrm{FDG}$ bone, $P<0.01$; ${ }^{\dagger}>$ Day 2 bone, $P<0.02$.
TABLE 3

Estimated Radiation Doses for ${ }^{64} \mathrm{Cu}$-DOTA-Trastuzumab*,

\begin{tabular}{lcc}
\hline Organ & $\begin{array}{c}\text { Equivalent or } \\
\text { effective dose per } \\
\text { unit injected activity } \\
(\mathrm{mSv} / \mathrm{MBq})\end{array}$ & $\begin{array}{c}\text { Equivalent or } \\
\text { effective dose per } \\
\text { PET examination } \\
(\mathrm{mSv})^{\ddagger}\end{array}$ \\
\hline Heart wall & 0.16 & 71 \\
\hline Kidneys & 0.09 & 42 \\
Liver & 0.12 & 53 \\
Red marrow & 0.04 & 17 \\
Spleen & 0.10 & 45 \\
Whole body & 0.02 & 10 \\
\hline Effective dose & 0.03 & 12 \\
\hline
\end{tabular}

${ }^{*}$ Trastuzumab protein dose $=50 \mathrm{mg}$.

${ }^{\dagger}$ Calculations used averaged time-activity curves (8 patients) from our ${ }^{111} \mathrm{In}$-MxDTPA-trastuzumab study (9), which were normalized to averaged blood and organ uptake data from current ${ }^{64} \mathrm{Cu}$-DOTA-trastuzumab study (6 patients).

${ }^{\ddagger}$ Assumes ${ }^{64} \mathrm{Cu}$ injected activity $=450 \mathrm{MBq}$, average in current study.

Patient selection and scan design for the ${ }^{64} \mathrm{Cu}$-DOTA-trastuzumab PET examinations relied on prior ${ }^{18} \mathrm{~F}-\mathrm{FDG}$ scans. Tumor uptake and detection sensitivity were only modestly lower for ${ }^{64} \mathrm{Cu}$-DOTAtrastuzumab than for ${ }^{18} \mathrm{~F}$-FDG. Most lesions were positively visualized with both radiotracers, and 6 CT-positive tumors were detected with ${ }^{64} \mathrm{Cu}$-DOTA-trastuzumab and not with ${ }^{18} \mathrm{~F}$-FDG. Same-lesion maximum SUVs for ${ }^{64} \mathrm{Cu}$-DOTA-trastuzumab and ${ }^{18} \mathrm{~F}$ FDG were uncorrelated, and their ratios $\left({ }^{64} \mathrm{Cu}\right.$-DOTA-trastuzumab to ${ }^{18} \mathrm{~F}-\mathrm{FDG}$ ) varied by a factor of 22 (Supplemental Fig. 2). Tumor uptake of ${ }^{18}$ F-FDG reflects density of glycolytic activity, which in turn depends on viable cell density and tissue oxygenation status (19-21). In breast cancer, high tumor uptake of ${ }^{18} \mathrm{~F}-\mathrm{FDG}$ is generally correlated with tumor aggressiveness but not with overexpression of the HER2 oncogene c-erbB-2 (22). For ${ }^{64} \mathrm{Cu}$-DOTAtrastuzumab, the unproven assumption is that tumor uptake is closely related to HER2 density, which in turn is positively correlated with tumor growth rate and aggressiveness (23). However, the relationship between uptake and HER2 expression may be confounded by factors such as blood clearance and vascular permeability. Because glycolysis and HER2 expression are independently related to tumor aggressiveness, the observed lack of correlation between same-tumor uptake of ${ }^{64} \mathrm{Cu}$-DOTA-trastuzumab and ${ }^{18} \mathrm{~F}$ FDG suggests that combining the 2 measurements may be useful in predicting patient outcomes.

The procedures used in this study were well tolerated. There were no unexpected toxicities associated with the trastuzumab or ${ }^{64} \mathrm{Cu}$-DOTA-trastuzumab administrations. Two patients had assay results that might indicate low-level anti-antibody responses after the ${ }^{64} \mathrm{Cu}$-DOTA-trastuzumab procedure. However, both patients had positive pre- ${ }^{64} \mathrm{Cu}$-DOTA-trastuzumab baseline assays and intermittently positive assays thereafter. This suggests positivity resulted from something other than the ${ }^{64} \mathrm{Cu}$-DOTA-trastuzumab procedure, such as prior treatment with trastuzumab or the presence of circulating antigen (i.e., HER2 extracellular domain) in the serum, a possibility that we are currently evaluating.

Estimated radiation doses for ${ }^{64} \mathrm{Cu}$-DOTA-trastuzumab (Table 3) are moderate, compared with ${ }^{18} \mathrm{~F}$-FDG and other imaging procedures with radiolabeled antibodies. For the mean administered ac- 
tivity in this study (450 MBq) and a 50-mg trastuzumab dose, estimated effective dose and maximum organ (heart wall) equivalent dose for ${ }^{64} \mathrm{Cu}$-DOTA-trastuzumab are 12 and $71 \mathrm{mSv}$, respectively. ${ }^{18} \mathrm{~F}$-FDG has effective and critical organ (bladder wall) equivalent doses of 11 and $72 \mathrm{mSv}$, respectively, for the typical injected activity of $555 \mathrm{MBq}(15 \mathrm{mCi})$ (24). Monoclonal antibodies labeled with ${ }^{111}$ In incur effective and critical organ (spleen and liver) equivalent doses of approximately 40 and $200 \mathrm{mSv}$, respectively, for the typical injected activity of $185 \mathrm{MBq}(5 \mathrm{mCi})$ (24). Dijkers et al. estimated a radiation dose (presumably effective dose) of $18 \mathrm{mSv}$ from a $37-\mathrm{MBq}(1-\mathrm{mCi})$ injection of ${ }^{89} \mathrm{Zr}$ trastuzumab (10).

\section{CONCLUSION}

We have shown that, in patients with HER2-positive metastatic breast cancer, tumors rapidly accumulate ${ }^{64} \mathrm{Cu}$-DOTA-trastuzumab to high concentrations, thus supporting both detection and measurement of tumor uptake by $1 \mathrm{~d}$ after injection. The rapid uptake, supplemented by prior knowledge of tumor location afforded by ${ }^{18}$ F-FDG PET/CT, makes ${ }^{64} \mathrm{Cu}$-DOTA-trastuzumab effective for surveying disseminated disease despite the limited half-life of ${ }^{64} \mathrm{Cu}$. We have confirmed that a trastuzumab dose of $50 \mathrm{mg}$ provides a ${ }^{64} \mathrm{Cu}$-DOTA-trastuzumab biodistribution favorable for tumor imaging. This study demonstrates that ${ }^{64} \mathrm{Cu}$-DOTA-trastuzumab $\mathrm{PET} / \mathrm{CT}$ is a practical and acceptably safe procedure in patients with metastatic breast cancer.

We will next broaden the study to include patients with metastatic breast cancer classified as HER2-negative on prescan biopsy and thus correlate tumor uptake of ${ }^{64} \mathrm{Cu}$-DOTA-trastuzumab with HER2 expression. Beyond that, we envision using ${ }^{64} \mathrm{Cu}-\mathrm{DOTA}-$ trastuzumab PET/CT to individualize treatment regimens that include trastuzumab- and other HER2-directed therapies.

\section{DISCLOSURE}

The costs of publication of this article were defrayed in part by the payment of page charges. Therefore, and solely to indicate this fact, this article is hereby marked "advertisement" in accordance with 18 USC section 1734 . This work was supported by the Department of Defense (grant 1024511) and by the National Cancer Institute of the National Institutes of Health under grant number P30CA033572. The content is solely the responsibility of the authors and does not necessarily represent the official views of the National Institutes of Health. No other potential conflict of interest relevant to this article was reported.

\section{ACKNOWLEDGMENTS}

Jose Reyes, CNMT, performed the PET/CT scans. Blood samples were assayed for radioactivity and anti-trastuzumab immune response by Nicole Bowles, BA, and Jing Guo, BS, respectively. The production of ${ }^{64} \mathrm{Cu}$ at Washington University School of Medicine is supported by the Department of Energy.

\section{REFERENCES}

1. Wolff AC, Hammond MEH, Schwartz JN, et al. American Society of Clinical Oncology/College of American Pathologists guideline recommendations for human epidermal growth factor receptor 2 testing in breast cancer. J Clin Oncol. 2007;25:118-145.
2. Hurvitz SA, Hu Y, O'Brien N, Finn RS. Current approaches and future directions in the treatment of HER2-positive breast cancer. Cancer Treat Rev. 2013;39:219-229.

3. Paik S, Kim C, Wolmark N. HER2 status and benefit from adjuvant trastuzumab in breast cancer. $N$ Engl J Med. 2008;358:1409-1411.

4. Perez EA, Suman VJ, Davidson NE, et al. HER2 testing by local, central, and reference laboratories in specimens from the North Central Cancer Treatment Group N9831 intergroup adjuvant trial. J Clin Oncol. 2006;24:3032-3038.

5. Simon R, Nocito A, Hübscher T, et al. Patterns of HER2/neu amplification and over-expression in primary and metastatic breast cancer. J Natl Cancer Inst. 2001; 93:1141-1146.

6. Zidan J, Dashkovsky I, Stayerman C, Basher W, Cozacov C, Hadary A. Comparison of HER-2 overexpression in primary breast cancer and metastatic sites and its effect on biological targeting therapy of metastatic disease. Br J Cancer. 2005;93:552-556.

7. Lindström LS, Karlsson E, Wilking UM, et al. Clinically used breast cancer markers such as estrogen receptor, progesterone receptor, and human epidermal growth factor receptor 2 are unstable throughout tumor progression. J Clin Oncol. 2012;30:2601-2608.

8. Perik PJ, Lub-De Hooge MN, Gietema JA, et al. Indium-111-labeled trastuzumab scintigraphy in patients with human epidermal growth factor receptor 2positive metastatic breast cancer. J Clin Oncol. 2006;24:2276-2282.

9. Wong JYC, Raubitschek A, Yamauchi D, et al. A pretherapy biodistribution and dosimetry study of indium-111-radiolabeled trastuzumab in patients with human epidermal growth factor receptor 2-overexpressing breast cancer. Cancer Biother Radiopharm. 2010;25:387-394.

10. Dijkers EC, Oude Munnink TH, Kosterink JG, et al. Biodistribution of ${ }^{89} \mathrm{Zr}$ trastuzumab and PET imaging of HER2-positive lesions in patients with metastatic breast cancer. Clin Pharmacol Ther. 2010;87:586-592.

11. Lub-de Hooge MN, Kosterink JGW, Perik PJ, et al. Preclinical characterization of ${ }^{111}$ In-DTPA-trastuzumab. Br J Pharmacol. 2004;143:99-106.

12. Li L, Bading J, Yazaki PJ, et al. A versatile bifunctional chelate for radiolabeling humanized anti-CEA antibody with In-111 and Cu-64 at either thiol or amino groups: PET imaging of CEA-positive tumors with whole antibodies. Bioconjug Chem. 2008;19:89-96.

13. Bading JR, Ng TSC, Jacobs RE, Raubitschek A, Colcher D. ${ }^{64} \mathrm{Cu}-\mathrm{DOTA}-H e r-$ ceptin: radio-synthesis and initial evaluation in vivo [abstract]. $\mathrm{J} \mathrm{Nucl} \mathrm{Med}$. 2009;50(suppl 2):404P.

14. Patt YZ, Lamki LM, Haynie TP, et al. Improved tumor localization with increasing dose of indium-111-labeled anti-carcinoembryonic antigen monoclonal antibody ZCE-025 in metastatic colorectal cancer. J Clin Oncol. 1988;6:1220-1230.

15. Cheebsumon $P$, Yaqub $M$, van Velden FH, Hoekstra OS, Lammertsma AA, Boellaard R. Impact of $\left[{ }^{18} \mathrm{~F}\right] \mathrm{FDG}$ PET imaging parameters on automatic tumour delineation: need for improved tumour delineation methodology. Eur J Nucl Med Mol Imaging. 2011;38:2136-2144.

16. Hong S, Li J, Wang S. ${ }^{18}$ FDG PET-CT for diagnosis of distant metastases in breast cancer patients: a meta-analysis. Surg Oncol. 2013;22:139-143.

17. O'Donoghue JA, Smith-Jones PM, Humm JL, et al. ${ }^{124}$ I-huA33 antibody uptake is driven by A33 antigen concentration in tissues from colorectal cancer patients imaged by immuno-PET. J Nucl Med. 2011;52:1878-1885.

18. Costantini DL, Chan C, Cai Z, Vallis KA, Reilly RM. ${ }^{111}$ In-labeled trastuzumab (Herceptin) modified with nuclear localization sequences (NLS): an Auger electronemitting radiotherapeutic agent for HER2/neu-amplified breast cancer. J Nucl Med. 2007; 48:1357-1368.

19. Wahl RL, Jacene H, Kasamon Y, Lodge MA. From RECIST to PERCIST: evolving considerations for PET response criteria in solid tumors. J Nucl Med. 2009;50:122S-150S.

20. Bos R, van der Hoeven JJM, van der Wall E, et al. Biologic correlates of ${ }^{18}$ Fluorodeoxyglucose uptake in human breast cancer measured by positron emission tomography. J Clin Oncol. 2002;20:379-387.

21. Minn H, Clavo AC, Wahl RL. Influence of hypoxia on tracer accumulation in squamous-cell carcinoma: in vitro evaluation for PET imaging. Nucl Med Biol. 1996;23:941-946.

22. Groheux D, Giachetti S, Moretti JL, et al. Correlation of high ${ }^{18}$ F-FDG uptake to clinical, pathological and biological prognostic factors in breast cancer. Eur $J$ Nucl Med Mol Imaging. 2011;38:426-435.

23. Slamon DJ, Clark GM, Wong SG, Levin WJ, Ullrich A, McGuire WL. Human breast cancer: correlation of relapse and survival with amplification of the HER2/neu oncogene. Science. 1987;235:177-182.

24. International Commission on Radiological Protection. Radiation dose to patients from radiopharmaceuticals: addendum 3 to ICRP publication 53. ICRP Publication 106. Ann ICRP. 2008;38:1-197. 